\title{
The pattern of choosing dialysis modality and related mortality outcomes in Korea: a national population-based study
}

Hyung Jong Kim ${ }^{1}$, Jung Tak Park², Seung Hyeok Han², Tae-Hyun Yoo ${ }^{2}$, Hyeong-Cheon Park ${ }^{2}$, Shin-Wook Kang 2 , Kyoung Hoon Kim³ ${ }^{3}$ Dong-Ryeol Ryu ${ }^{4}$, and Hyunwook Kim²

${ }^{1}$ Department of Internal Medicine, CHA Bundang Medical Center, CHA University, Seongnam; ${ }^{2}$ Department of Internal Medicine, Yonsei University College of Medicine, Seoul; ${ }^{3}$ Department of Public Health, Korea University Graduate School, Seoul; ${ }^{4}$ Department of Internal Medicine, Tissue Injury Defense Research Center, Ewha Womans University School of Medicine, Seoul, Korea

Received: March 16, 2017

Revised : May 29, 2017

Accepted: June 14, 2017

\section{Correspondence to}

Hyunwook Kim, M.D.

Department of Internal Medicine, Gangnam Severance Hospital, Yonsei University College of Medicine, 211 Eonju-ro, Gangnam-gu, Seoul o6273, Korea

Tel: +82-2-2019-3310

Fax: $+82-2-3463-3882$

E-mail: bluejayway@naver.com
Background/Aims: Since comorbidities are major determinants of modality choice, and also interact with dialysis modality on mortality outcomes, we examined the pattern of modality choice according to comorbidities and then evaluated how such choices affected mortality in incident dialysis patients.

Methods: We analyzed 32,280 incident dialysis patients in Korea. Patterns in initial dialysis choice were assessed by multivariate logistic regression analyses. Multivariate Poisson regression analyses were performed to evaluate the effects of interactions between comorbidities and dialysis modality on mortality and to quantify these interactions using the synergy factor.

Results: Prior histories of myocardial infarction $(p=0.031)$, diabetes $(p=0.001)$, and congestive heart failure $(p=0.003)$ were independent factors favoring the initiation with peritoneal dialysis (PD), but were associated with increased mortality with PD. In contrast, a history of cerebrovascular disease and 1-year increase in age favored initiation with hemodialysis (HD) and were related to a survival benefit with $\mathrm{HD}(p<0.001$, both). While favoring initiation with HD, having Medical $\operatorname{Aid}(p=0.001)$ and male gender $(p=0.047)$ were related to increased mortality with HD. Furthermore, although the severity of comorbidities did not influence dialysis modality choice, mortality in incident PD patients was significantly higher compared to that in HD patients as the severity of comorbidities increased ( $p$ for trend < 0.001).

Conclusions: Some comorbidities exerted independent effects on initial choice of dialysis modality, but this choice did not always lead to the best results. Further analyses of the pattern of choosing dialysis modality according to baseline comorbid conditions and related consequent mortality outcomes are needed.

Keywords: Renal dialysis; Peritoneal dialysis; Comorbidity; Mortality

\section{INTRODUCTION}

The number of end-stage renal disease (ESRD) patients requiring dialysis treatment has been continuously and rapidly increasing over the past few decades. Although there is little controversy that kidney transplantation is the best renal replacement treatment for ESRD, there are many obstacles to its timely initiation, including a shortage of donated organs, cultural differences, or poor socioeconomic status. Consequently, more than $95 \%$ of 
incident ESRD patients are forced to initiate dialysis treatment with hemodialysis (HD) or peritoneal dialysis (PD) [1]. In this situation, choice of dialysis modality is particularly important and known to be influenced by various factors such as geography, patient characteristics and preferences, and medical conditions [2]. Among these factors, the patients' underlying comorbidities are found to be the major independent determinants of dialysis modality choice [3]. Furthermore, these preexisting comorbidities showed significant interactions with dialysis modality on patients' outcomes $[4,5]$. In this light, despite the relevance of surveying the nationwide patterns of dialysis modality choice according to underlying comorbidities and examining associated clinical outcomes, only a few studies have addressed this relationship [6].

We previously reported that, although it is being improved in recent years [7], PD is generally inferior to $\mathrm{HD}$ as an initial dialysis modality in Korea in terms of overall survival, and this is largely accounted for by the higher incidence of all-cause mortality, non-fatal acute myocardial infarction (MI), and vascular complications requiring target vessel revascularization $[8,9]$ in incident PD patients. This finding was in contrast to recent studies of large-scale registry data, but the reasons for this inferiority of PD could not be adequately explained [10-12]. Therefore, in this study, we approached this issue first by assessing the pattern of modality choice for incident dialysis patients according to baseline comorbid conditions in Korea, and then evaluated how such choice affected mortality outcomes by calculating the synergy factor (SF) from the interaction analysis based on a multiplicative scale between the various comorbid conditions and dialysis modality [13].

\section{METHODS}

\section{Data source and study population}

We used the Korean Health Insurance Review and Assessment Service (HIRA) database. The detail of HIRA database organization was described elsewhere [9]. The Institutional Review Board at the HIRA approved our use of the database for this study (IRB No. 3159, 2012).

We initially screened all Korean incident dialysis patients who started dialysis therapy between January
1, 2005 and December 31, 2008. Among them, we only included patients who initiated dialysis treatment during the study period and remained on chronic dialysis therapy for at least 3 months. We excluded patients who were younger than 18 years. Finally, our study included 32,280 eligible patients. Further, we included both the patients who have National Health Insurance and those who have Medical Aid as primary insurance; Medical Aid is a public assistance program for poor individuals who are under the National Basic Livelihood Security System in Korea as a part of the social welfare programs [14]. In all analyses for comparisons between dialysis modalities, an intention-to-treat principle was adopted.

The comorbid conditions of the participants were identified by screening the medical records during the year before the initiation of dialysis therapy. The list of comorbidities was determined and scored based on the suggestions of Charlson et al. [15], and ICD-10 (International Classification of Diseases 1oth revision) codes were used according to the algorithms proposed by Quan et al. [16].

\section{Measurements and analyses of outcomes}

The primary clinical endpoint of interest for our study was all-cause mortality. For assessing event-free survival, we considered the dialysis modality at day 90 to be the initial dialysis modality and used day 90 as the starting point (day o). That is, the patients analyzed in this study were left-censored for the first event-free 90 days after dialysis initiation, and they were right-censored at December 31, 2009.

\section{Statistical analysis}

In comparison analyses, baseline parameters were compared using an independent $t$ test for continuous variables and Pearson chi-square test for categorical variables. There was no missing data on baseline characteristics of participants and their outcomes. Crude incidence rates of mortality were calculated by dividing the number of deaths by the person-years of follow-up, expressed as deaths per 1,000 patient-years; confidence intervals (CIs) were estimated based on a Poisson distribution.

Multivariable-adjusted logistic regression analyses were conducted to estimate odds ratios for the likelihood of choosing an initial dialysis modality between two modalities according to the presence or absence of 
particular comorbid conditions. Cox proportional hazard regression analyses were performed to evaluate the increase in the absolute mortality risk associated with an initial dialysis modality according to the baseline comorbid conditions.

In addition, to assess the degree of change in the relative risk (RR) for mortality via effect modification between dialysis modality and various baseline comorbid conditions, interaction terms were constructed and entered into multivariable-adjusted Poisson regression analyses to test the statistical significance of these interactions on mortality. Furthermore, we quantified the degrees of these interactions in terms of SF with $95 \%$ CIs, based on a multiplicative scale. In brief, SF was calculated from the adjusted RRs. We first estimated the RRs for factor $x_{1}$ alone $\left(R R_{1}\right)$, factor $x_{2}$ alone $\left(R R_{2}\right)$, and both factors combined $\left(\mathrm{RR}_{12}\right)$. SF was then defined as the ratio of the observed $R R$ for both factors combined and presented as $\mathrm{SF}=\mathrm{RR}_{12} /\left(\mathrm{RR}_{1} \times \mathrm{RR}_{2}\right)$. Thus, if $\mathrm{SF}>1$, this was interpreted as a positive interaction (a synergistic increase in the risk) between the two risk factors [13].

All statistical tests were evaluated using a two-tailed $95 \%$ CI, and $p<0.05$ was considered statistically significant. All statistical analyses were conducted using the statistical package SAS version 9.3 (SAS Institute Inc., Cary, NC, USA).

\section{RESULTS}

\section{Baseline characteristics}

A total of 32,280 eligible patients who initiated dialysis therapy between January 1, 2005 and December 31, 2008, were analyzed. When comparing patients who initiated dialysis therapy with $\mathrm{PD}$ versus $\mathrm{HD}$, incident $\mathrm{PD}$ patients were significantly more likely to be younger and female, and more likely to have National Health Insurance (vs. Medical Aid) than incident HD patients. While the prevalence of diabetes and chronic pulmonary disease (CPD) was similar, the overall distribution of comorbid conditions was significantly different between patients on PD and those on HD (Table 1). The severity of comorbidities assessed by the Charlson comorbidity index (CCI) was also significantly different, and a high comorbidity burden (CCI $\geq 3$ ) was significantly more prevalent in incident HD patients than in incident PD patients. During the median follow-up of 24.5 months (range, 3.1 to 59.0) and 67,541 patient-years, 7,820 deaths were observed, for an overall estimated death rate of 116 deaths (95\% CI, 113 to 118) per 1,000 patient-years.

\section{Comparison of the absolute mortality risk between PD and $\mathrm{HD}$ according to baseline comorbid conditions}

As shown in Fig. 1, we performed Cox proportional regression analyses and found that overall absolute mortality risk adjusted for all baseline covariates listed in Table 1 was significantly $23 \%$ higher in the patients initiating dialysis with PD than those with HD. Then, we explored whether this trend was also apparent in various subgroups according to baseline comorbid conditions, and found that the patients initiating dialysis with PD generally have a significant higher absolute risk for mortality than those with HD across all subgroups. However, some exceptions were noted; there were no significant differences in mortality rates between PD and HD in the patients with age $<60$ years, Medical Aid as their primary insurance, liver disease, cancer, or no comorbidity.

\section{The pattern of choosing dialysis modality according to each comorbid condition and related mortality outcomes in all incident dialysis patients}

First, we examined which baseline comorbid conditions were independently associated with the choice of a particular dialysis modality in all incident dialysis patients. After adjusting for all baseline conditions listed in Table 1, prior histories of MI, congestive heart failure (CHF), and diabetes were independently associated with a higher likelihood of receiving PD as an initial dialysis modality, whereas prior histories of cancer, cerebrovascular disease (CVD), and liver disease; Medical Aid as primary insurance; male gender; and a 1-year increase in age were independently associated with a higher likelihood of receiving HD as an initial dialysis modality (Table 2, Fig. 2, black bars).

Next, as shown by $p$ values of interaction analyses in Fig. 1, we found that the relative degrees of risk for mortality increased by PD were significantly modified by the presence or absence of a particular comorbid condition. Therefore, we further examined the effects of these interactions between the independent determinants of initial modality choice and various baseline comorbid conditions on mortality (Table 3, Fig. 2, red bars). Among 
Table 1. Baseline characteristics of the participants

\begin{tabular}{|c|c|c|c|c|}
\hline Variable & $\begin{array}{l}\text { All incident dialysis patients } \\
\qquad(\mathrm{n}=32,280)\end{array}$ & $\begin{array}{l}\text { Incident PD patients } \\
(\mathrm{n}=7,881)\end{array}$ & $\begin{array}{l}\text { Incident HD patients } \\
(\mathrm{n}=24,399)\end{array}$ & $\begin{array}{c}p \text { value } \\
(\mathrm{PD} \text { vs. } \mathrm{HD})\end{array}$ \\
\hline Age, yr & $57.0 \pm 13.8$ & $54.2 \pm 13.6$ & $57.8 \pm 13.8$ & $<0.001$ \\
\hline$\geq 60$ & $14,706(45 \cdot 6)$ & $2,968(37.7)$ & $11,738(48.1)$ & $<0.001$ \\
\hline Sex, female (vs. male) & $13,337(41.3)$ & $3,443(43.7)$ & $9,894(40.6)$ & $<0.001$ \\
\hline NHI (vs. MA) & $27,859(86.3)$ & $6,898(87.5)$ & $20,961(85.9)$ & $<0.001$ \\
\hline Diabetes mellitus & $16,186(50.1)$ & $3,996(50.7)$ & $12,190(50.0)$ & 0.251 \\
\hline \multicolumn{5}{|l|}{ Comorbidities other than DM } \\
\hline Myocardial infarction & $1,164(3.6)$ & $367(4.7)$ & $797(3 \cdot 3)$ & $<0.001$ \\
\hline Congestive heart failure & $4,752(14.7)$ & $1,269(16.1)$ & $3,483(14 \cdot 3)$ & $<0.001$ \\
\hline Peripheral artery disease & $1,908(5.9)$ & $424(5.4)$ & $1,484(6.1)$ & 0.022 \\
\hline Cerebrovascular disease & $4,038(12.5)$ & $830(10.5)$ & $3,208(13.1)$ & $<0.001$ \\
\hline Chronic pulmonary disease & $5,221(16.2)$ & $1,233(15.6)$ & $3,988(16.3)$ & 0.142 \\
\hline Peptic ulcer disease & $4,709(14.6)$ & $1,072(13.6)$ & $3,637(14.9)$ & 0.004 \\
\hline Liver disease & $3,460(10.7)$ & $780(9.9)$ & $2,680(11.0)$ & 0.007 \\
\hline Cancer & $1,968(6.1)$ & $321(4.1)$ & $1,647(6.8)$ & $<0.001$ \\
\hline CCI & $2.2 \pm 1.9$ & $2.2 \pm 1.9$ & $2.3 \pm 1.9$ & $<0.001$ \\
\hline \multicolumn{5}{|l|}{ Distribution of CCI scores } \\
\hline o & $7,618(23.6)$ & $1,918(24 \cdot 3)$ & $5,700(23.4)$ & 0.076 \\
\hline 1 & $5,424(16.8)$ & $1,336(17.0)$ & $4,088(16.8)$ & 0.684 \\
\hline 2 & $5,731(17.8)$ & $1,464(18.6)$ & $4,267(17.5)$ & 0.028 \\
\hline$\geq 3$ & $13,507(41.8)$ & $3,163(40.1)$ & $10,344(42.4)$ & $<0.001$ \\
\hline
\end{tabular}

Values are presented as mean \pm SD or number (\%).

PD, peritoneal dialysis; HD, hemodialysis; NHI, National Health Insurance; MA, Medical Aid; DM, diabetes mellitus; CCI, Charlson comorbidity index.

the independent determinants that led to the choice of $\mathrm{PD}$ as an initial modality, prior histories of MI, diabetes, and CHF showed a significant positive interaction with the initiation of dialysis with PD; that is, increased the risk of mortality when initiating dialysis treatment with PD. On the other hand, having Medical Aid as primary insurance and male gender were significant independent determinants of dialysis initiation with $\mathrm{HD}$, and these choice patterns were eventually associated with a synergistically increased risk of mortality. In contrast, a history of CVD and a 1-year increase in age, which were significant independent determinants of dialysis initiation with HD, were associated with worse survival with PD (better survival with HD) (Table 3, Fig. 2).
The pattern of choosing dialysis modality according to each comorbid condition and related mortality outcomes in all incident dialysis patients $<60$ years

When stratifying by age, we found that, in patients younger than 60 years, prior histories of MI, CHF, CPD, and diabetes were independently associated with a higher likelihood of receiving PD as an initial dialysis modality, whereas having Medical Aid as their primary insurance, male gender, a history of cancer, and a 1-year increase in age were independently associated with a higher likelihood of receiving HD as an initial dialysis modality (Supplementary Table 1).

The consequences of this choice are shown in Supplementary Table 2 and indicate that, among the determinants independently associated with the choice of PD as an initial modality, prior histories of MI and diabetes synergistically increased the risk of mortality when 


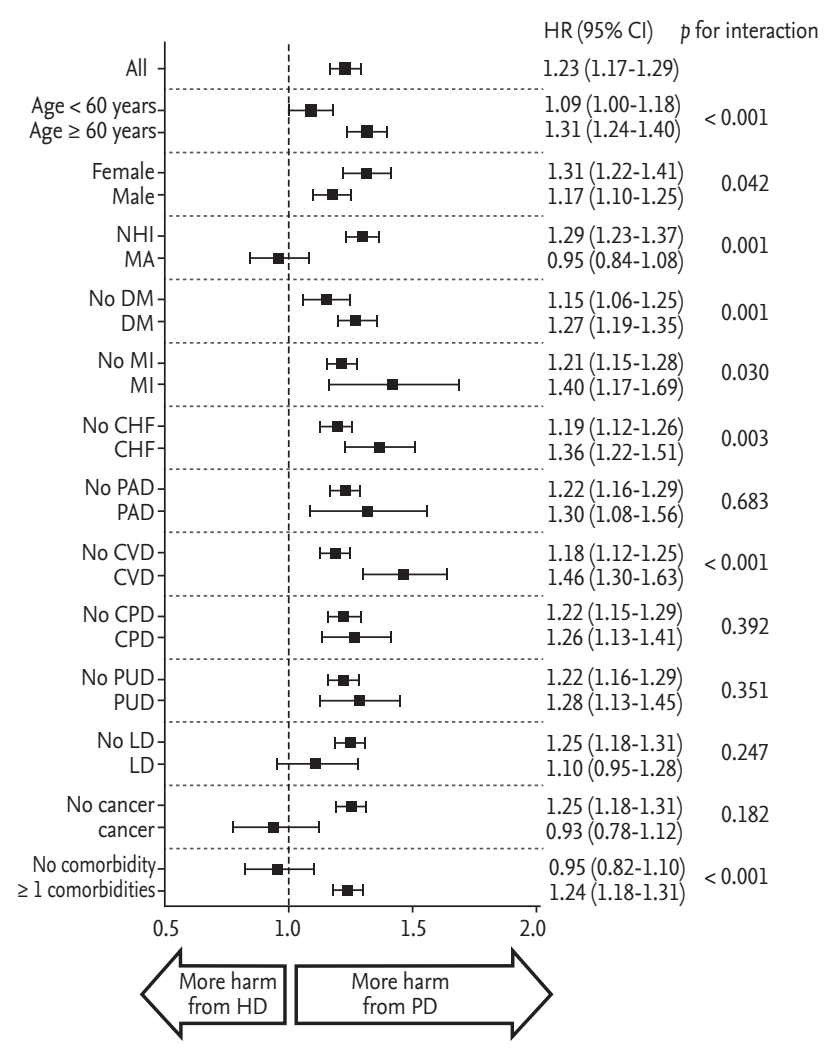

Figure 1. Comparisons of multivariable-adjusted mortality risks between peritoneal dialysis (PD) and hemodialysis (HD) by subgroups (adjusted hazard ratios [HRs] and 95\% confidence intervals [CIs] for mortality by multivariable-adjusted Cox proportional hazard regression analyses). NHI, National Health Insurance; MA, Medical Aid; DM, diabetes mellitus; MI, myocardial infarction; $\mathrm{CHF}$, congestive heart failure; PAD, peripheral artery disease; CVD, cerebrovascular disease; CPD, chronic pulmonary disease; PUD, peptic ulcer disease; LD, liver disease.

starting dialysis treatment with PD. On the other hand, among the determinants independently associated with the choice of HD as an initial modality, having Medical Aid as their primary insurance synergistically increased the risk of mortality when starting dialysis treatment with HD. In contrast, a 1-year increase in age, which was a significant independent determinant of dialysis initiation with $\mathrm{HD}$, was associated with worse survival with PD (better survival with HD) (Supplementary Table 2).

The pattern of choosing dialysis modality according to each comorbid condition and related mortality outcomes in all incident dialysis patients $\geq 60$ years

In patients aged 60 years or older, prior histories of MI and CHF were independently related to the choice of PD as an initial dialysis modality, whereas prior history of cancer, liver disease, or CVD; male gender; and a 1-year increase in age were independently associated with a higher likelihood of receiving HD as an initial dialysis modality (Supplementary Table 3). Among the significant determinants that led to the choice of PD as an initial modality, a prior history of CHF was significantly related to the synergistically increased risk of mortality when combined with the initiation of dialysis treatment on PD. On the other hand, among the significant determinants that led to the choice of HD as an initial modality, a prior history of cancer and male gender were significantly related to the synergistically increased risk of mortality when combined with the initiation of dialysis treatment on HD (Supplementary Table 4).

The pattern of choosing dialysis modality according to the severity and number of comorbidities and related mortality outcomes

Finally, we sought to evaluate the interaction effects between the pattern of modality choice and the severity and number of comorbidities on mortality outcomes. As shown in Table 4, Fig. 3, there was no significant difference in the odds of choice for either type of dialysis modality according to the severity of comorbidities assessed by CCI or the number of comorbidity ( $\geq$ 1 comorbidities vs. no comorbidity). However, looking at the consequences of such choice, we found that, as the severity and the number of comorbidities increased, the mortality risks of patients initiating dialysis therapy with PD were significantly increased compared to those initiating with $\mathrm{HD}$; this trend was evident even from a CCI score of 1 or higher $(p$ for trend $<0.001$ ) (Table 5 , Fig. 4).

\section{DISCUSSION}

In the current study, we found that some comorbid conditions were independently related to the choice of dialysis modality, and that this choice might not always lead to the best mortality outcome. Instead, such choices often synergistically increased mortality. To approach this issue is far from simple because preexisting comorbidity conditions are not the only determinant of dialysis 
Table 2. Multivariable-adjusted likelihood to choose dialysis modality according to baseline comorbid conditions in all incident dialysis patients

\begin{tabular}{|c|c|c|}
\hline Baseline comorbid condition & Odds ratio $(95 \% \mathrm{CI})^{\mathrm{a}}$ & $p$ value \\
\hline \multicolumn{3}{|c|}{ More likely to choose PD as an initial dialysis modality } \\
\hline Myocardial infarction ${ }^{\mathrm{b}}$ & $1.61(1.41-1.83)$ & $<0.001$ \\
\hline Congestive heart failure ${ }^{\mathrm{b}}$ & $1.20(1.11-1.29)$ & $<0.001$ \\
\hline Diabetes mellitus ${ }^{\mathrm{b}}$ & $1.13(1.07-1.19)$ & $<0.001$ \\
\hline Chronic pulmonary disease & $1.04(0.97-1.11)$ & 0.310 \\
\hline \multicolumn{3}{|c|}{ More likely to choose HD as an initial dialysis modality } \\
\hline Cancer & $1.49(1.32-1.69)$ & $<0.001$ \\
\hline MA (vs. NHI) $)^{b}$ & $1.22(1.13-1.32)$ & $<0.001$ \\
\hline Sex, male (vs. female) ${ }^{b}$ & $1.17(1.11-1.23)$ & $<0.001$ \\
\hline Cerebrovascular disease $^{c}$ & $1.14(1.05-1.23)$ & 0.003 \\
\hline Liver disease & $1.10(1.01-1.20)$ & 0.031 \\
\hline Peptic ulcer disease & $1.04(0.97-1.13)$ & 0.221 \\
\hline Peripheral artery disease & $1.04(0.93-1.16)$ & 0.543 \\
\hline Age (per 1-year increase) ${ }^{c}$ & $1.02(1.02-1.02)$ & $<0.001$ \\
\hline
\end{tabular}

CI, confidence interval; PD, peritoneal dialysis; HD, hemodialysis; MA, Medical Aid; NHI, National Health Insurance.

${ }^{a}$ Adjusted odds ratios and 95\% CIs by multivariable-adjusted logistic regression analysis for choosing each dialysis modality.

${ }^{\mathrm{b}}$ The pattern of choice for dialysis modality in this condition consequently increased the mortality risk.

${ }^{\circ}$ The pattern of choice for dialysis modality in this condition consequently conferred the survival benefit.

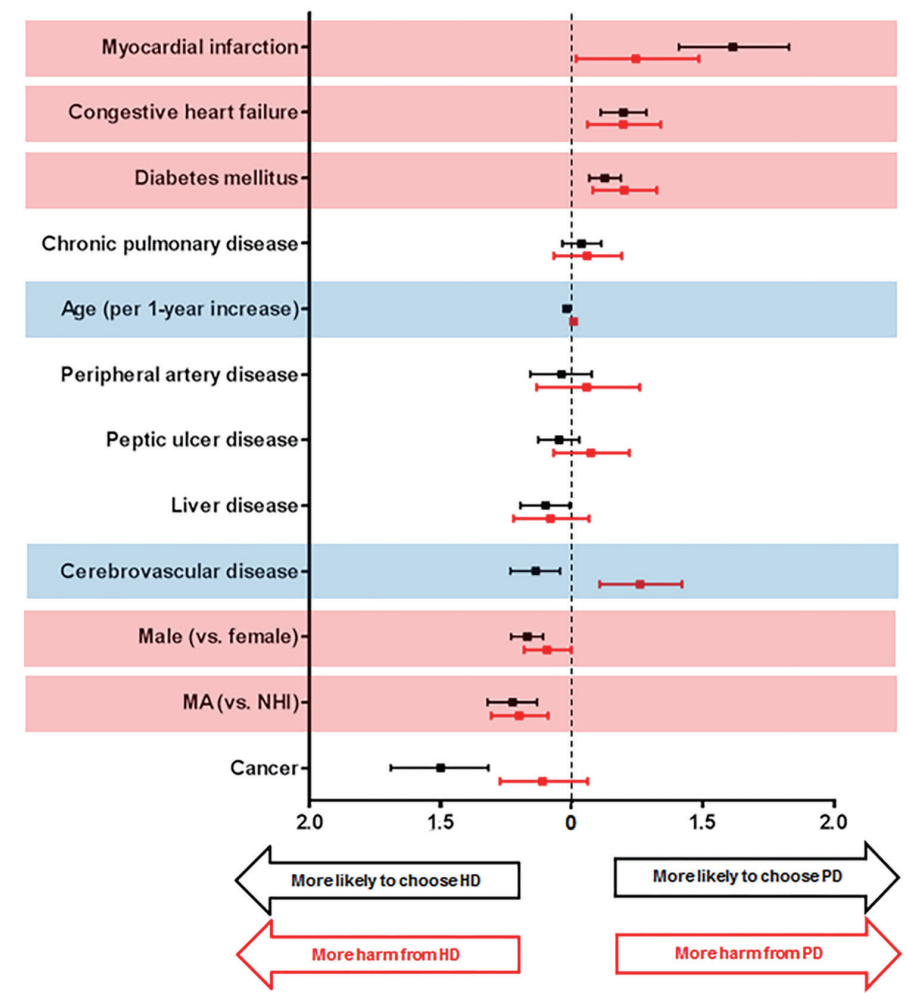

Figure 2. The pattern of choosing dialysis modality according to baseline comorbid conditions and related mortality outcomes. Black bars: multivariable-adjusted likelihood to choose dialysis modality according to baseline comorbid conditions in all incident dialysis patients (adjusted odds ratios and $95 \%$ confidence intervals [CIs] by multivariable-adjusted logistic regression analysis for choosing each dialysis modality). Red bars: multivariable-adjusted likelihood to experience more harm from one modality than from the other modality in all incident dialysis patients (adjusted synergy factors and $95 \%$ CIs based on a multiplicative interaction between initial dialysis modality and baseline comorbid conditions on mortality). Red shaded area: the pattern of choice for dialysis modality in this condition consequently increased the mortality risk $(p<0.05)$. Blue shaded area: the pattern of choice for dialysis modality in this condition consequently conferred the survival benefit ( $p$ $<0.05)$. MA, Medical Aid; NHI, National Health Insurance; $\mathrm{HD}$, hemodialysis; $\mathrm{PD}$, peritoneal dialysis. 
Table 3. Multivariable-adjusted likelihood to experience more harm from one modality than from the other modality in all incident dialysis patients

\begin{tabular}{|c|c|c|}
\hline Baseline comorbid condition & $\mathrm{SF}(95 \% \mathrm{CI})^{\mathrm{a}}$ & $p$ value \\
\hline \multicolumn{3}{|c|}{ More harm from PD as an initial dialysis modality } \\
\hline Cerebrovascular disease ${ }^{\mathrm{b}}$ & $1.25(1.11-1.42)$ & $<0.001$ \\
\hline Myocardial infarction ${ }^{\mathrm{c}}$ & $1.23(1.02-1.48)$ & 0.031 \\
\hline Diabetes mellitus ${ }^{\mathrm{c}}$ & $1.20(1.08-1.32)$ & 0.001 \\
\hline Congestive heart failure ${ }^{c}$ & $1.09(1.06-1.34)$ & 0.003 \\
\hline Peptic ulcer disease & $1.07(0.93-1.22)$ & 0.346 \\
\hline Chronic pulmonary disease & $1.05(0.93-1.19)$ & 0.394 \\
\hline Peripheral artery disease & $1.05(0.87-1.26)$ & 0.641 \\
\hline Age (per 1-year increase) ${ }^{b}$ & $1.01(1.00-1.01)$ & $<0.001$ \\
\hline \multicolumn{3}{|c|}{ More harm from HD as an initial dialysis modality } \\
\hline $\mathrm{MA}\left(\right.$ vs. NHI) ${ }^{\mathrm{c}}$ & $1.26(1.10-1.44)$ & 0.001 \\
\hline Cancer & $1.14(0.94-1.37)$ & 0.182 \\
\hline Sex, male (vs. female) ${ }^{\mathrm{c}}$ & $1.11(1.00-1.22)$ & 0.047 \\
\hline Liver disease & $1.10(0.94-1.28)$ & 0.249 \\
\hline \multicolumn{3}{|c|}{$\begin{array}{l}\text { SF, synergy factor; CI, confidence interval; PD, peritoneal dialysis; HD, hemodialysis; MA, Medical Aid; Nl } \\
\text { Insurance. } \\
{ }^{a} \text { Adjusted SFs and } 95 \% \text { CIs based on a multiplicative interaction between initial dialysis modality and base } \\
\text { ditions on mortality. } \\
{ }^{{ }^{2}} \text { The pattern of choice for dialysis modality in this condition consequently conferred the survival benefit. } \\
{ }^{c} \text { The pattern of choice for dialysis modality in this condition consequently increased the mortality risk. }\end{array}$} \\
\hline
\end{tabular}

Table 4. Multivariable-adjusted likelihood to choose PD as an initial dialysis modality according to the number and severity of comorbidities measured by CCI in all incident dialysis patients

\begin{tabular}{|c|c|c|}
\hline Baseline comorbid condition & Odds ratio $(95 \% \mathrm{CI})^{\mathrm{a}}$ & $p$ value \\
\hline \multicolumn{3}{|c|}{ More likely to choose PD as an initial dialysis modality } \\
\hline With no comorbidity & 1.00 (reference) & - \\
\hline With $\geq 1$ comorbidity & $1.04(0.97-1.12)$ & 0.307 \\
\hline \multicolumn{3}{|c|}{ The severity of comorbidities measured by CCI } \\
\hline o & 1.00 (reference) & - \\
\hline 1 & $1.04(0.96-1.13)$ & 0.347 \\
\hline 2 & $1.09(0.99-1.20)$ & 0.088 \\
\hline$\geq 3$ & $0.96(0.87-1.06)$ & 0.438 \\
\hline
\end{tabular}

PD, peritoneal dialysis; CCI, Charlson comorbidity index; CI, confidence interval.

${ }^{a}$ Adjusted odds ratios and 95\% CIs by multivariable-adjusted logistic regression analysis for choosing each dialysis modality.

modality in both physicians and patients, but various other factors are also involved.

During the study period, patients with diabetes, MI, or CHF had a significantly higher likelihood of being regarded as proper candidates for initiating dialysis therapy with $\mathrm{PD}$, but all of these comorbid conditions syner- gistically increased the intrinsic mortality risk posed by PD per se. On the other hand, even though Medical Aid as primary insurance and male gender led to avoidance of PD as an initial dialysis modality, those conditions were found to significantly decrease the mortality risk conferred by PD per se (increased mortality risk with 
HD). A preferred implementation of PD for patients with diabetes has also been documented in several previous reports $[3,17-19]$. This finding can be explained by the belief in the potential advantages of PD over HD for maintaining more stable hemodynamic status, particu-

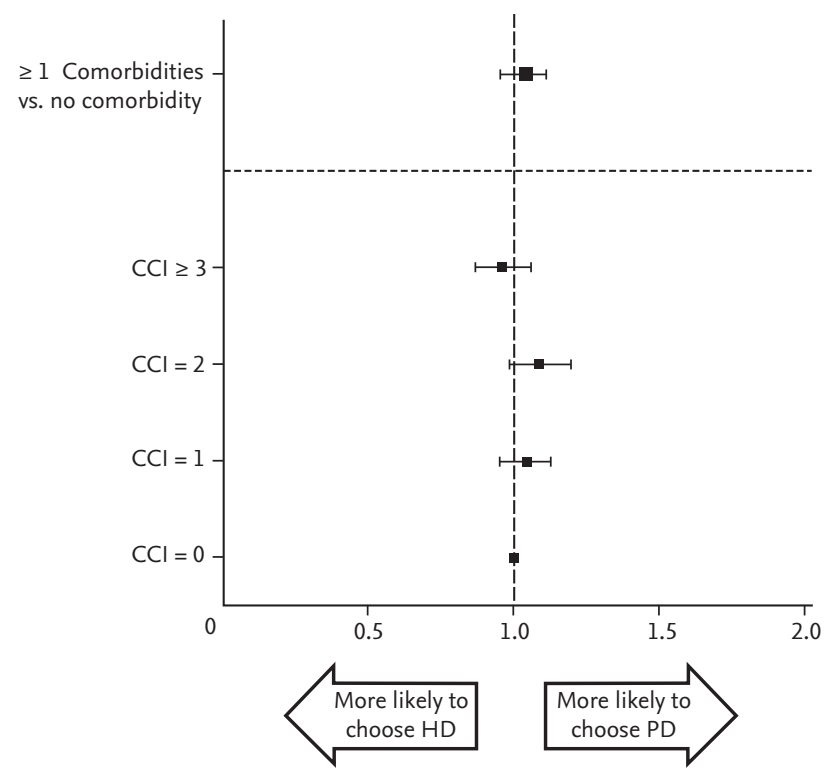

Figure 3. Multivariable-adjusted likelihood to choose peritoneal dialysis (PD) as an initial dialysis modality according to the number and severity of comorbidities measured by Charlson comorbidity index (CCI) in all incident dialysis patients (adjusted odds ratios and 95\% confidence intervals by multivariable-adjusted logistic regression analysis for choosing each dialysis modality). HD, hemodialysis. larly under diabetes-related autonomic dysfunction and the absence of requirements for usable vascular access, which is difficult to achieve due to diabetes-related atherosclerotic calcification. However, contrary to this view but in accordance with our study, a large proportion of studies to date indicate that PD is associated with worse outcomes than HD when performed in diabetic patients [20-22]. Whether diabetes per se should be recognized as a contraindication to the use of PD remains highly controversial and far from being definitively answered [23].

In addition, a considerable number of studies have suggested that PD has possible advantages compared to HD in patients with underlying cardiac dysfunction. In particular, patients with medical treatment-resistant CHF have few therapeutic options other than heart transplantation, surgical plasty, or extracorporeal ultrafiltration. In such cases, short-term use of PD provides symptomatic relief and improvement of various cardiac indices $[24,25]$. In a similar way, PD is regarded as also having potential benefits for patients with coronary artery disease. Partly due to less abrupt hemodynamic disturbance than HD, PD is associated with a low probability of development of myocardial stunning represented by regional wall motion abnormalities, which are frequently observed in HD [26]. However, these inferences are based on small-scale observations that are neither adequately validated nor were evidenced in large-scale studies; rather, dialysis initiation with PD is associated

Table 5. Multivariable-adjusted likelihood to experience more harm with PD as an initial dialysis modality according to the number and severity of comorbidities measured by CCI in all incident dialysis patients

\begin{tabular}{lcc}
\hline Baseline comorbid condition & SF $(95 \% \mathrm{CI})^{\mathrm{a}}$ & $p$ value \\
\hline More harm from PD as an initial dialysis modality & & - \\
With no comorbidity & 1.00 (reference) & $<0.001$ \\
With $\geq 1$ comorbidity & $1.40(1.20-1.64)$ & - \\
The severity of comorbidities measured by CCI & 1.00 (reference) & 0.011 \\
0 & $1.30(1.06-1.60)$ & $<0.001$ \\
1 & $1.48(1.23-1.78)$ & $<0.001$ \\
2 & $1.46(1.24-1.72)$ & $<0.001$ \\
\hline for trend & - & $<$ \\
\hline
\end{tabular}

PD, peritoneal dialysis; CCI, Charlson comorbidity index; SF, synergy factor; CI, confidence interval.

${ }^{a}$ Adjusted SFs and $95 \%$ CIs based on a multiplicative interaction between initial dialysis modality and the number/severity of comorbidities on mortality.

${ }^{\mathrm{b}_{\mathrm{T}}}$ The linear trend in an increase in the mortality risk with PD vs. hemodialysis (reference) from CCI $=0$ to CCI $\geq 3$. 


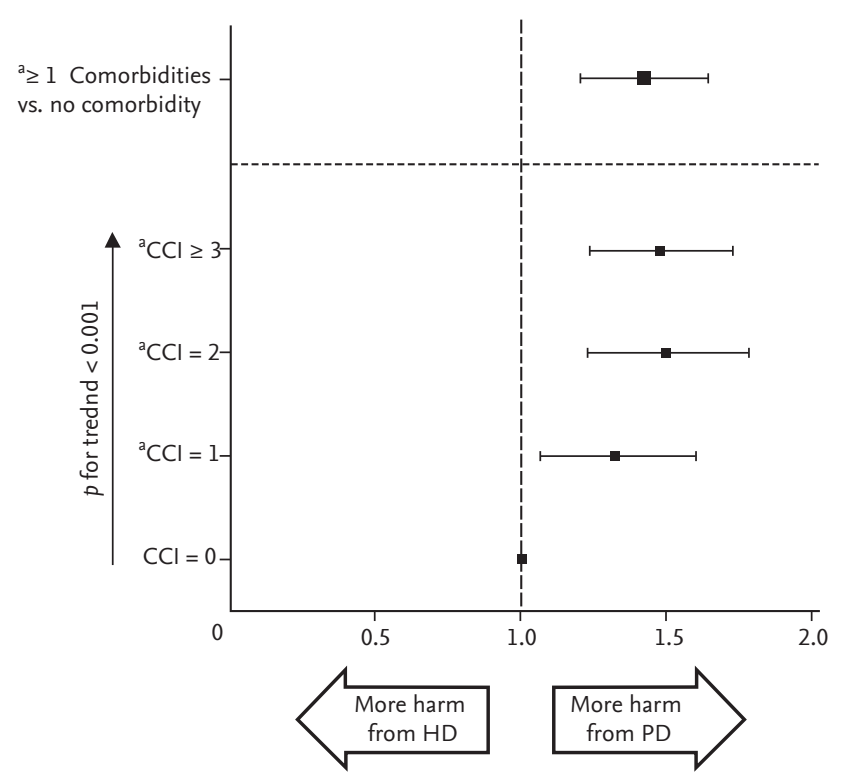

Figure 4. Multivariable-adjusted likelihood to experience more harm with peritoneal dialysis (PD) as an initial dialysis modality according to the number and severity of comorbidities measured by Charlson comorbidity index (CCI) in all incident dialysis patients (adjusted synergy factors and $95 \%$ confidence intervals based on a multiplicative interaction between initial dialysis modality and the number/ severity of comorbidities on mortality). HD, hemodialysis. ${ }^{a}$ The increases in the number of comorbidities ( $\geq 1$ comorbidities vs. no comorbidity) and CCI scores significantly associated with the increase in mortality when accompanied by PD as an initial dialysis modality $(p<0.05)$.

with significantly higher mortality risk than with $\mathrm{HD}$ in incident ESRD patients with underlying CHF [5,27] or coronary artery disease [4]. Supporting these findings, a substantial number of studies have suggested that PD might be related to a greater atherogenic vascular risk than HD given a higher prevalence of dyslipidemia [28], a higher level of atherogenic lipoprotein(a) [29], and a higher prevalence of overhydration-related high blood pressure [30,31]. Continuous exposure to high glucose load induced by PD fluid might also contribute to PD-related cardiac compromise, possibly mediated through hyperinsulinemia with insulin resistance, hyperleptinemia, or inflammation with oxidative stress [32,33]. On the other hand, older patients or those with prior CVD were more likely to choose $\mathrm{HD}$ as an initial dialysis modality, and this trend in choice consequently conferred a survival benefit. Consistent with this, the relative advantages of $\mathrm{HD}$ over PD in elderly patients have been shown in previous reports [17,34], particularly in those with diabetes or longer dialysis vintage [35]. The cause of higher mortality with PD versus HD in elderly patients is not clear. However, it should be noted that cardiovascular mortality, as the most common cause of general ESRD patients, cannot solely account for this; rather, as the age of ESRD patients increases, noncardiovascular causes of mortality were more predominant than cardiovascular causes [20]. This pattern is especially apparent in PD-treated patients with diabetes, female gender, and advanced age [34]. In addition, partly due to functional impairments in handling PD equipments including PD solution exchange, and in performing PD-related selfcare, patients with CVD are not only less likely to initiate dialysis treatment with PD but also have potential disadvantages that can lead to PD-related adverse outcomes. In a long-term follow-up study of the patients who experienced a stroke, the most common cause of death was cardiovascular disease, with a rate of $67.4 \%$ [36]. This high atherosclerotic burden prior to and post stroke might not only be further complicated by the abovementioned metabolic derangements induced by $\mathrm{PD}$, but also independently associated with an increased risk of $\mathrm{PD}$ peritonitis, which is an independent predictor of mortality [37,38].

When analyzed by age ( $\geq 60$ years vs. $<60$ years), we can observe that, as age increases, the number of comorbid conditions as well as the degree of risk increase independently related to the choice of a particular dialysis modality was decreased, suggesting that age itself might serve as the primary prognostic factor. However, underlying cardiac morbidities such as MI or CHF was consistently synergistically increased the risk of mortality when initiating dialysis treatment with $\mathrm{PD}$, irrespective of age groups.

In this study, we also found that the number or severity of comorbidities was not a determinant of the dialysis modality choice. However, increased mortality was observed in a significant linear trend when patients with a higher comorbidity initiated dialysis with PD compared to HD ( $p$ for trend < 0.001 ). The distribution of the degree of comorbidities measured by CCI or other parameters between incident patients on HD and PD is not consistent across studies. It has been reported that, in Canada, the United States, and Europe, patients with a higher comorbidity level tend to start dialysis therapy 
with HD instead of PD [6,18,19,39], whereas, in Columbia, incident PD patients have a significantly higher degree of comorbidity than HD patients [40]. How dialysis modalities modify the mortality risk according to the degree of comorbidities has also been investigated in a few studies. In agreement with our findings, studies investigating the European ERA-EDTA (The European Renal Association-European Dialysis and Transplant Association) and Australia and New Zealand registries commonly showed the superiority of PD over HD for survival in patients with no comorbidity, but this effect disappears as the number of comorbidities increases $[6,41]$. Also in a large Taiwanese cohort stratified by CCI score, the patients belonging to the group with the highest CCI score had a significant 21\% higher mortality risk with PD than with HD [42].

When focusing on HD, we found that patients who were male and had Medical Aid as their primary insurance were significantly more likely to start dialysis treatment with $\mathrm{HD}$, but this choice was associated with an increase in mortality risk. The trend that incident ESRD patients on PD were of higher socioeconomic status than those on HD was in accordance with previous studies $[18,39]$. This difference may result from several parameters that are difficult to measure, such as hygiene status, accessibility to medical service, or reimbursement factors [43].

We should note the limitations of this study and necessary precautions in interpreting the results. First, since the comorbidities listed in this study were assessed by reviewing the diagnosis codes claimed, the possibility of underestimation or oversimplification of the comorbidity burden or of missing the effects of other important conditions not addressed in this study could not be excluded. Second, due to the limitations of the dataset, we could not access either the causes of death or the information on dialysis-related factors potentially affecting patients' mortality such as HD adequacy, nutritional status, infection rates, or other non-conventional risk factors. Fourth, and most importantly, we focused only on the relative degrees of advantage or disadvantage of one modality versus the other modality using SF based on multiplicative interaction analyses. Therefore, caution is required when interpreting the results.

In conclusion, we found that some types and severity of comorbidities exerted independent effects on the choice of dialysis modality, but this choice did not always lead to the best outcomes. Based on our observations, the overall worse outcomes of incident PD patients compared to HD patients during the study period might result from, at least in part, more frequent use of PD in the patients with prior MI, CHF, diabetes or with a higher degree of comorbidity. To improve the survival rate of dialysis patients, further intensive investigation of the pattern of choosing dialysis modality according to baseline comorbid and other conditions, and reviews of the related consequences on mortality outcomes are warranted.

\section{KEY MESSAGE}

1. Some types and severity of comorbidities exerted independent effects on the choice of dialysis modality, but this choice did not always lead to the best outcomes.

2. The overall worse outcomes of incident peritoneal dialysis (PD) patients compared to hemodialysis patients during the study period (between January 1, 2005 and December 31, 2009) might result from more frequent use of $\mathrm{PD}$ in the patients with prior myocardial infarction, congestive heart failure, diabetes or with a higher degree of comorbidity.

3. Further intensive investigation of the pattern of choosing dialysis modality according to baseline comorbid and other conditions, and reviews of the related consequences on mortality outcomes are warranted to improve the survival rate of dialysis patients.

\section{Conflict of interest}

No potential conflict of interest relevant to this article was reported.

\section{REFERENCES}

1. ESRD in the United States: an overview of USRDS annual data report volume 2. Am J Kidney Dis 2015;66(1 Suppl 1):S79-S92.

2. van de Luijtgaarden MW, Jager KJ, Stel VS, et al. Global 
differences in dialysis modality mix: the role of patient characteristics, macroeconomics and renal service indicators. Nephrol Dial Transplant 2013;28:1264-1275.

3. Stack AG. Determinants of modality selection among incident US dialysis patients: results from a national study. J Am Soc Nephrol 2002;13:1279-1287.

4. Ganesh SK, Hulbert-Shearon T, Port FK, Eagle K, Stack AG. Mortality differences by dialysis modality among incident ESRD patients with and without coronary artery disease. J Am Soc Nephrol 2003;14:415-424.

5. Stack AG, Molony DA, Rahman NS, Dosekun A, Murthy B. Impact of dialysis modality on survival of new ESRD patients with congestive heart failure in the United States. Kidney Int 2003;64:1071-1079.

6. van de Luijtgaarden MW, Noordzij M, Stel VS, et al. Effects of comorbid and demographic factors on dialysis modality choice and related patient survival in Europe. Nephrol Dial Transplant 2011;26:2940-2947.

7. Ryu JH, Kim H, Kim KH, et al. Improving survival rate of Korean patients initiating dialysis. Yonsei Med J 2015;56:666-675.

8. Kim H, Kim KH, Park K, et al. A population-based approach indicates an overall higher patient mortality with peritoneal dialysis compared to hemodialysis in Korea. Kidney Int 2014;86:991-1000.

9. Kim H, Kim KH, Ahn SV, et al. Risk of major cardiovascular events among incident dialysis patients: a Korean national population-based study. Int J Cardiol 2015;198:95101.

10. Mehrotra R, Chiu YW, Kalantar-Zadeh K, Bargman J, Vonesh E. Similar outcomes with hemodialysis and peritoneal dialysis in patients with end-stage renal disease. Arch Intern Med 2011;171:110-118.

11. van de Luijtgaarden MW, Jager KJ, Segelmark M, et al. Trends in dialysis modality choice and related patient survival in the ERA-EDTA Registry over a 20 -year period. Nephrol Dial Transplant 2016;31:120-128.

12. Noordzij M, Jager KJ. Patient survival on dialysis in Korea: a different story? Kidney Int 2014;86:877-880.

13. Cortina-Borja M, Smith AD, Combarros O, Lehmann DJ. The synergy factor: a statistic to measure interactions in complex diseases. BMC Res Notes 2009;2:105.

14. Kim JH, Lee KS, Yoo KB, Park EC. The differences in health care utilization between Medical Aid and health insurance: a longitudinal study using propensity score matching. PLoS One 2015;10:eo119939.
15. Charlson ME, Pompei P, Ales KL, MacKenzie CR. A new method of classifying prognostic comorbidity in longitudinal studies: development and validation. J Chronic Dis 1987;40:373-383.

16. Quan H, Sundararajan V, Halfon P, et al. Coding algorithms for defining comorbidities in ICD-9-CM and ICD-10 administrative data. Med Care 2005;43:1130-1139.

17. Vonesh EF, Snyder JJ, Foley RN, Collins AJ. The differential impact of risk factors on mortality in hemodialysis and peritoneal dialysis. Kidney Int 2004;66:2389-2401.

18. Miskulin DC, Meyer KB, Athienites NV, et al. Comorbidity and other factors associated with modality selection in incident dialysis patients: the CHOICE Study. Choices for Healthy Outcomes in Caring for End-Stage Renal Disease. Am J Kidney Dis 2002;39:324-336.

19. Yeates K, Zhu N, Vonesh E, Trpeski L, Blake P, Fenton S. Hemodialysis and peritoneal dialysis are associated with similar outcomes for end-stage renal disease treatment in Canada. Nephrol Dial Transplant 2012;27:3568-3575.

20. de Jager DJ, Grootendorst DC, Jager KJ, et al. Cardiovascular and noncardiovascular mortality among patients starting dialysis. JAMA 2009;302:1782-1789.

21. Liem YS, Wong JB, Hunink MG, de Charro FT, Winkelmayer WC. Comparison of hemodialysis and peritoneal dialysis survival in The Netherlands. Kidney Int 2007;71:153-158.

22. Vonesh EF, Snyder JJ, Foley RN, Collins AJ. Mortality studies comparing peritoneal dialysis and hemodialysis: what do they tell us? Kidney Int Suppl 2006;(103):S3-S11.

23. Couchoud C, Bolignano D, Nistor I, et al. Dialysis modality choice in diabetic patients with end-stage kidney disease: a systematic review of the available evidence. Nephrol Dial Transplant 2015;30:310-320.

24. Gotloib L, Fudin R, Yakubovich M, Vienken J. Peritoneal dialysis in refractory end-stage congestive heart failure: a challenge facing a no-win situation. Nephrol Dial Transplant 2005;20 Suppl 7:viiz2-viiz6.

25. Koch M, Haastert B, Kohnle M, et al. Peritoneal dialysis relieves clinical symptoms and is well tolerated in patients with refractory heart failure and chronic kidney disease. Eur J Heart Fail 2012;14:530-539.

26. Selby NM, McIntyre CW. Peritoneal dialysis is not associated with myocardial stunning. Perit Dial Int 2011;31:2733.

27. Sens F, Schott-Pethelaz AM, Labeeuw M, Colin C, Villar E; REIN Registry. Survival advantage of hemodialysis 
relative to peritoneal dialysis in patients with end-stage renal disease and congestive heart failure. Kidney Int 2011;80:970-977.

28. Liu J, Rosner MH. Lipid abnormalities associated with end-stage renal disease. Semin Dial 2006;19:32-40.

29. Kronenberg F, Konig P, Neyer U, et al. Multicenter study of lipoprotein(a) and apolipoprotein(a) phenotypes in patients with end-stage renal disease treated by hemodialysis or continuous ambulatory peritoneal dialysis. J Am Soc Nephrol 1995;6:110-120.

30. Konings CJ, Kooman JP, Schonck M, et al. Fluid status, blood pressure, and cardiovascular abnormalities in patients on peritoneal dialysis. Perit Dial Int 2002;22:477487.

31. Plum J, Schoenicke G, Kleophas W, et al. Comparison of body fluid distribution between chronic haemodialysis and peritoneal dialysis patients as assessed by biophysical and biochemical methods. Nephrol Dial Transplant 2001;16:2378-2385.

32. Stenvinkel P, Lindholm B, Lonnqvist F, Katzarski K, Heimburger O. Increases in serum leptin levels during peritoneal dialysis are associated with inflammation and a decrease in lean body mass. J Am Soc Nephrol 2000;11:1303-1309.

33. Holmes C, Mujais S. Glucose sparing in peritoneal dialysis: implications and metrics. Kidney Int Suppl 2006;(103):S104-S109.

34. Collins AJ, Hao W, Xia H, et al. Mortality risks of peritoneal dialysis and hemodialysis. Am J Kidney Dis 1999;34:1065-1074.

35. Han SS, Park JY, Kang S, et al. Dialysis modality and mortality in the elderly: a meta-analysis. Clin J Am Soc Nephrol 2015;10:983-993.

36. Bronnum-Hansen H, Davidsen M, Thorvaldsen P; Danish MONICA Study Group. Long-term survival and causes of death after stroke. Stroke 2001;32:2131-2136.

37. Nadeau-Fredette AC, Johnson DW, Hawley CM, et al. Center-specific factors associated with peritonitis risk-a multi-center registry analysis. Perit Dial Int 2016;36:509518.

38. Fried LF, Bernardini J, Johnston JR, Piraino B. Peritonitis influences mortality in peritoneal dialysis patients. J Am Soc Nephrol 1996;7:2176-2182.

39. Jaar BG, Coresh J, Plantinga LC, et al. Comparing the risk for death with peritoneal dialysis and hemodialysis in a national cohort of patients with chronic kidney disease. Ann Intern Med 2005;143:174-183.

40. Sanabria M, Munoz J, Trillos C, et al. Dialysis outcomes in Colombia (DOC) study: a comparison of patient survival on peritoneal dialysis vs hemodialysis in Colombia. Kidney Int Suppl 2008;(108):S165-S172.

41. McDonald SP, Marshall MR, Johnson DW, Polkinghorne KR. Relationship between dialysis modality and mortality. J Am Soc Nephrol 2009;20:155-163.

42. Chang YK, Hsu CC, Hwang SJ, et al. A comparative assessment of survival between propensity score-matched patients with peritoneal dialysis and hemodialysis in Taiwan. Medicine (Baltimore) 2012;91:144-151.

43. Just PM, de Charro FT, Tschosik EA, Noe LL, Bhattacharyya SK, Riella MC. Reimbursement and economic factors influencing dialysis modality choice around the world. Nephrol Dial Transplant 2008;23:2365-2373. 
Supplementary Table 1. Multivariable-adjusted likelihood to choose dialysis modality according to baseline comorbid conditions in incident dialysis patients aged less than 60 years

\begin{tabular}{|c|c|c|}
\hline Baseline comorbid condition & Odds ratio $(95 \% \mathrm{CI})^{\mathrm{a}}$ & $p$ value \\
\hline \multicolumn{3}{|c|}{ More likely to choose PD as an initial dialysis modality } \\
\hline Myocardial infarction ${ }^{b}$ & $1.33(1.06-1.67)$ & 0.015 \\
\hline Congestive heart failure & $1.17(1.05-1.29)$ & 0.003 \\
\hline Chronic pulmonary disease & $1.15(1.04-1.27)$ & 0.008 \\
\hline Diabetes mellitus $^{\mathrm{b}}$ & $1.13(1.05-1.21)$ & 0.001 \\
\hline \multicolumn{3}{|c|}{ More likely to choose HD as an initial dialysis modality } \\
\hline MA (vs. NHI) ${ }^{b}$ & $1.28(1.16-1.40)$ & $<0.001$ \\
\hline Sex, male (vs. female) & $1.23(1.15-1.31)$ & $<0.001$ \\
\hline Cancer & $1.22(1.03-1.45)$ & 0.019 \\
\hline Peripheral artery disease & $1.06(0.89-1.26)$ & 0.507 \\
\hline Liver disease & $1.06(0.95-1.17)$ & 0.311 \\
\hline Cerebrovascular disease & $1.05(0.92-1.19)$ & 0.452 \\
\hline Peptic ulcer disease & $1.03(0.93-1.14)$ & 0.559 \\
\hline Age (per 1-year increase) ${ }^{c}$ & $1.02(1.01-1.02)$ & $<0.001$ \\
\hline
\end{tabular}

CI, confidence interval; PD, peritoneal dialysis; HD, hemodialysis; MA, Medical Aid; NHI, National Health Insurance.

${ }^{a}$ Adjusted odds ratios and $95 \%$ CIs by multivariable-adjusted logistic regression analysis for choosing each dialysis modality.

${ }^{\mathrm{b}}$ The pattern of choice for dialysis modality in this condition consequently increased the mortality risk.

'The pattern of choice for dialysis modality in this condition consequently conferred the survival benefit. 
Supplementary Table 2. Multivariable-adjusted likelihood to experience more harm from one modality than from the other modality in incident dialysis patients aged less than 60 years

\begin{tabular}{|c|c|c|}
\hline Baseline comorbid condition & $\mathrm{SF}(95 \% \mathrm{CI})^{\mathrm{a}}$ & $p$ value \\
\hline \multicolumn{3}{|c|}{ More harm from PD as an initial dialysis modality } \\
\hline Myocardial infarction ${ }^{\mathrm{b}}$ & $1.52(1.00-2.29)$ & 0.049 \\
\hline Cerebrovascular disease & $1.46(1.15-1.83)$ & 0.001 \\
\hline Diabetes mellitus ${ }^{\mathrm{b}}$ & $1.23(1.04-1.47)$ & 0.018 \\
\hline Congestive heart failure & $1.20(0.97-1.47)$ & 0.094 \\
\hline Cancer & $1.09(0.83-1.44)$ & 0.532 \\
\hline Age (per 1-year increase) ${ }^{c}$ & $1.01(1.00-1.02)$ & 0.016 \\
\hline Sex, male (vs. female) & $1.01(0.85-1.20)$ & 0.889 \\
\hline \multicolumn{3}{|c|}{ More harm from HD as an initial dialysis modality } \\
\hline $\mathrm{MA}\left(\right.$ vs. NHI) ${ }^{\mathrm{b}}$ & $1.42(1.17-1.73)$ & 0.001 \\
\hline Chronic pulmonary disease & $1.08(0.87-1.35)$ & 0.489 \\
\hline Liver disease & $1.02(0.82-1.28)$ & 0.838 \\
\hline Peripheral artery disease & $1.01(0.69-1.47)$ & 0.977 \\
\hline Peptic ulcer disease & $1.00(0.79-1.26)$ & 0.990 \\
\hline
\end{tabular}

SF, synergy factor; CI, confidence interval; PD, peritoneal dialysis; HD, hemodialysis; MA, Medical Aid; NHI, National Health Insurance.

${ }^{a}$ Adjusted SFs and 95\% CIs based on a multiplicative interaction between initial dialysis modality and baseline comorbid conditions on mortality.

${ }^{\mathrm{b}}$ The pattern of choice for dialysis modality in this condition consequently increased the mortality risk.

${ }^{\circ}$ The pattern of choice for dialysis modality in this condition consequently conferred the survival benefit. 
Supplementary Table 3. Multivariable-adjusted likelihood to choose dialysis modality according to baseline comorbid conditions in incident dialysis patients aged 60 years or older

\begin{tabular}{|c|c|c|}
\hline Baseline comorbid condition & Odds ratio $(95 \% \mathrm{CI})^{\mathrm{a}}$ & $p$ value \\
\hline \multicolumn{3}{|c|}{ More likely to choose PD as an initial dialysis modality } \\
\hline Myocardial infarction & $1.77(1.51-2.08)$ & $<0.001$ \\
\hline Congestive heart failure ${ }^{\mathrm{b}}$ & $1.23(1.11-1.29)$ & $<0.001$ \\
\hline Diabetes mellitus & $1.05(0.96-1.14)$ & 0.273 \\
\hline \multicolumn{3}{|c|}{ More likely to choose HD as an initial dialysis modality } \\
\hline Cancer $^{\mathrm{b}}$ & $1.87(1.55-2.25)$ & $<0.001$ \\
\hline Liver disease & $1.22(1.05-1.42)$ & 0.010 \\
\hline Cerebrovascular disease & $1.20(1.08-1.34)$ & 0.001 \\
\hline MA (vs. NHI) & $1.12(0.98-1.29)$ & 0.090 \\
\hline Sex, male (vs. female) ${ }^{b}$ & $1.09(1.00-1.18)$ & 0.041 \\
\hline Peptic ulcer disease & $1.07\left(0.95^{-1.19)}\right.$ & 0.272 \\
\hline Chronic pulmonary disease & $1.05\left(0.95^{-1.17)}\right.$ & 0.315 \\
\hline Age (per 1-year increase) & $1.04(1.03-1.05)$ & $<0.001$ \\
\hline Peripheral artery disease & $1.02(0.87-1.19)$ & 0.812 \\
\hline
\end{tabular}

CI, confidence interval; PD, peritoneal dialysis; HD, hemodialysis; MA, Medical Aid; NHI, National Health Insurance.

${ }^{a}$ Adjusted odds ratios and 95\% CIs by multivariable-adjusted logistic regression analysis for choosing each dialysis modality.

${ }^{\mathrm{b}}$ The pattern of choice for dialysis modality in this condition consequently increased the mortality risk. 
Supplementary Table 4. Multivariable-adjusted likelihood to experience more harm from one modality than from the other modality in incident dialysis patients aged 60 years or older

\begin{tabular}{|c|c|c|}
\hline Baseline comorbid condition & $\mathrm{SF}(95 \% \mathrm{CI})^{\mathrm{a}}$ & $p$ value \\
\hline \multicolumn{3}{|c|}{ More harm from PD as an initial dialysis modality } \\
\hline Congestive heart failure ${ }^{b}$ & $1.17(1.02-1.35)$ & 0.028 \\
\hline Cerebrovascular disease & $1.15(0.99-1.33)$ & 0.068 \\
\hline Diabetes mellitus & $1.15(1.01-1.30)$ & 0.034 \\
\hline Myocardial infarction & $1.13(0.91-1.40)$ & 0.267 \\
\hline Peripheral artery disease & $1.08(0.87-1.34)$ & 0.469 \\
\hline Peptic ulcer disease & $1.07(0.91-1.26)$ & 0.418 \\
\hline Chronic pulmonary disease & $1.06(0.91-1.22)$ & 0.462 \\
\hline \multicolumn{3}{|c|}{ More harm from HD as an initial dialysis modality } \\
\hline Cancer $^{\mathrm{b}}$ & $1.49(1.15-1.94)$ & 0.003 \\
\hline Liver disease & $1.16(0.93-1.45)$ & 0.175 \\
\hline Sex, male (vs. female) ${ }^{b}$ & $1.16(1.03-1.31)$ & 0.015 \\
\hline MA (vs. NHI) & $1.16(0.96-1.40)$ & 0.132 \\
\hline Age (per 1-year increase) & $1.01(1.00-1.02)$ & 0.316 \\
\hline
\end{tabular}

SF, synergy factor; CI, confidence interval; PD, peritoneal dialysis; HD, hemodialysis; MA, Medical Aid; NHI, National Health Insurance.

${ }^{a}$ Adjusted SFs and 95\% CIs based on a multiplicative interaction between initial dialysis modality and baseline comorbid conditions on mortality.

${ }^{\mathrm{b}}$ The pattern of choice for dialysis modality in this condition consequently increased the mortality risk. 